Methods Newly diagnosed 252 consecutive PD patients were included and followed as part of an ongoing PD registry. PD was diagnosed according to the United Kingdom brain bank diagnostic criteria. 79 PD patients fulfilled the DSM-IV criteria for major depression. The UPDRS motor score was checked at the best "on" period to assess the clinical severity of $\mathrm{PD}$. We compared the clinical data between depressive (DP, $\mathrm{n}=79$ ) and non-depressive (NDP, $\mathrm{n}=173$ ) groups.

Results The prevalence rate of depression in $\mathrm{PD}$ was $31.3 \%$ in this study. There was no difference in age (DP: $62.3 \pm 2.5$, NDP: $59.8 \pm 2.7 \mathrm{yrs}$ ), age of disease onset (DP: $52.0 \pm 3.7 \mathrm{yrs}$, NDP: $54.7 \pm 3.8 \mathrm{yrs}$ ), UPDRS motor scores (DP: $36.2 \pm 5.6$, NDP: $33.8 \pm 3.7$ ) and Hoehn and Yahr stage (DP: $3.34 \pm 0.52$, NDP: 2.91 \pm 0.63 ) between two groups.

Conclusion There was no significant difference in clinical features between DP and NDP groups in this study. These results suggest that depression in $\mathrm{PD}$ is not influenced the severity of motor symptoms and that non-dopaminergic neurotransmitters, such as norepinephrine and acetylcholine, at least associated with the pathophysiology of depression in PD.

\section{SP1-46 SCHOOL-BASED INTERVENTION TO PROMOTE HEALTHY NUTRITION AND PHYSICAL ACTIVITY IN SOUSSE, TUNISIA}

doi:10.1136/jech.2011.142976n.23

M M Jihene, ${ }^{*}$ H Imed, G Rafika, B A Imene, B Mylene, G Hassen. Farhat Hached Hospital, Sousse, Tunisia

Throughout the world, and particularly in Tunisia, children are becoming overweight and obese at progressively younger ages. Our purpose was to evaluate the effects of a school intervention program to promote healthy nutrition and physical activity among adolescents, in terms of behaviours and intention.

Methods It was a quasi experimental intervention study with two groups: control and intervention group with pre-post evaluation of nutrition and physical activity intention and behaviour in each group. The target population was composed with students aged $12-16$ years schooled in colleges of Sousse in Tunisia. To evaluate the intervention, a sampling was used to include 2200 students who participated to the questionnaire. All the students of intervention group received a standardised program with information about healthy nutrition and physical activity. An Arabic pre-tested and auto-administered questionnaire was used to assess nutrition and physical activity intention and behaviour before and after the intervention.

Results The intervention group's posttest intention and behaviour were significantly higher than the control group's posttest. Concerning "healthy behaviours", in the intervention group, children improved significantly their vegetable intake. It passed from $45.4 \%$ to $56.8 \%(\mathrm{p}<0.001)$. The proportion of children who practice 5 days per week physical activity for at least $30 \mathrm{~min}$ a day increased significantly from $23 \%$ to $44 \%$ ( $p<0.001)$.

Conclusion School based intervention to promote healthy diet and physical activity is benefit and important at the middle age to prevent obesity and cardiovascular disease risk factors.

\section{SP1-47 ASSOCIATIONS BETWEEN PERSISTENT ORGANIC POLLUTANTS (POPS) IN VISCERAL AND SUBCUTANEOUS ADIPOSE TISSUE AND INSULIN RESISTANCE}

doi:10.1136/jech.2011.142976n.24

${ }^{1} Y$ M Lee, ${ }^{*}{ }^{1} S$ A Kim, ${ }^{2}$ S G Kim, ${ }^{3}$ K K Lee, ${ }^{1}$ B Y Chun, ${ }^{1} \mathrm{D}$ H Lee. ${ }^{1}$ Department of Preventive Medicine, School of Medicine, Kyungpook National University, Daegu, Republic of Korea; ' 2 Department of Surgery, School of Medicine, Kyungpook National
University, Daegu, Republic of Korea; ${ }^{3}$ Department of Internal Medicine, School of Medicine, Kyungpook National University, Daegu, Republic of Korea

Introduction Persistent Organic Pollutants (POPs) is recently linked to insulin resistance and type 2 diabetes. Although POPs are mostly bioaccumulated in adipose tissues, most studies have measured serum concentration of POPs because of difficulties of collecting adipose tissues. This study was performed 1) to compare patterns of concentrations of POPs between visceral adipose tissue (VAT) and subcutaneous adipose tissue (SAT), and 2) to investigate associations of insulin resistance with concentrations of POPs in VAT or SAT.

Methods We collected both VAT and SAT from 50 patients who underwent abdominal surgery and analysed 14 organochlorinated pesticides (OCPs) and 19 Polychlorinated biphenyls (PCBs). Insulin resistance was estimated using homeostasis model assessment method (HOMA-IR).

Results Concentrations of OCPs and PCBs among VAT and SAT were highly correlated, but absolute concentrations of PCBs in VAT were 3-4 times higher than those of SAT. As concentrations of p,p'-DDT, p,p'-DDD, cis-nonachlordane, trans-nonachlordane, PCB28, PCB105, and PCB118 in VAT or SAT increased, HOMA-IR significantly increased. The risk of elevated HOMA-IR $(>50$ th percentile) was 5 to 10 times higher among subjects in the 3rd tertile of these POPs compared with those in the 1st tertile. Although here are some differences depending on individual POP, the positive associations between POPs and HOMA-IR were generally more obvious in VAT than SAT. Also, the extent of macrophage infiltration in VAT was positively associated with concentrations of POPs in VAT, not SAT.

Conclusion The current study strongly suggested that some POPs accumulated in VAT may be involved in the development of insulin resistance.

\section{SP1-48 REDUCTIONS IN DENTAL CARIES IN 3-YEAR-0LD CHILDREN IN GREATER GLASGOW AND CLYDE}

doi:10.1136/jech.2011.142976n.25

${ }^{1}$ A D McMahon, * ${ }^{2} Y$ Blair, ${ }^{2} \mathrm{D}$ R McCall, ${ }^{1} \mathrm{~L}$ M D Macpherson. ${ }^{1}$ Glasgow University Dental School, Glasgow, Scotland, UK; ${ }^{2}$ NHS Greater Glasgow and Clyde, Glasgow, Scotland, UK

The objective of this study was to examine dental inspection data from 3-year-old children in Greater Glasgow and Clyde, Scotland, over 4 years to assess oral health trends during a period of implementation of a national child dental health improvement programme (Childsmile). Dental inspections of 3-year-old children in Greater Glasgow and Clyde were undertaken in the academic years 2006/7, 2007/8 (the baseline years), 2008/9 and 2009/10 (post intervention). The number of decayed, missing and filled teeth was calculated $\left(\mathrm{d}_{3} \mathrm{mft}\right)$, together with the percentage $\mathrm{d}_{3} \mathrm{mft}>0$. The study also examined the effect of socioeconomic status using the Scottish Index of Multiple Deprivation (SIMD). In total, 10022 children were inspected (19\% of the population). The weighted percentage of children with caries experience was $26 \%$ in $2006 / 7,25 \%$ (2007/8), reducing to $18 \%$ (2007/8) and 17\% (2009/ 10). When compared to the first baseline year of $2006 / 7$, the OR was 0.91 for 2007/8 (0.79-1.06, $p=0.221), 0.63$ for 2008/9 $(0.55-0.72, \mathrm{p}<0.001)$, and 0.50 for $2009 / 10(0.43-0.58, \mathrm{p}<0.001)$. The weighted mean $\mathrm{d}_{3} \mathrm{mft}$ was 1.1 in $2006 / 7,1.0$ in $2007 / 8$ $(p=0.869), 0.6$ in $2008 / 9(p<0.001)$ and 0.4 in $2009 / 10(p<0.001)$. Caries reductions were seen in all socioeconomic groups. This study demonstrates that it is possible to impact upon the prevalence and morbidity of dental caries across the socioeconomic spectrum in a population. The dental health of young children in the NHS Greater Glasgow and Clyde Board area has improved significantly in recent years. 